\title{
STUDYING SOME CHARACTERISTICS AND PARAMETERS OF ARGON GLOW DISCHARGE PLASMA USING HOLLOW ANODE DIAMETER
}

\author{
Sh. Kh. Al-Hakary*, Diyar A. Bleej, Esraa A. Mohammed Saeed, Suzan M. Haji \& Sahme A. Hussaine
}

Dept. of Physics, Faculty of Science, University of Zakho, Kurdistan Region, Iraq - (shamo.awsi@uoz.edu.krd)

\begin{abstract}
:
Hollow anode argon glow discharge plasma has been investigated experimentally at different argon gas pressure from $\left(2.2 \times 10^{-2}\right.$ to 3$)$ mbar at constant discharge current. A sufficient high voltage has been applied among the electrodes to obtain breakdown. Firstly, we studied the influence of hollow anode diameter on the breakdown voltage and Paschens law. The inner diameters of hollow anodes used in our work were $(10,15,20,25,30,35$, and 40$) \mathrm{mm}$. Secondly under the same conditions we extended our study to measure some plasma parameters in the negative glow region using direct current argon glow discharge. The temperature and density of electrons in the negative glow were measured using double probes. From the $\left(I_{p}-V_{p}\right)$ characteristics of double probes, we obtained plasma parameters by using computer MATLAB program. The results showed that the measured Pashence's curve closes to the well-known theoretical Pashence's law. The breakdown voltage and its minimum value decreased with increasing the hollow anode diameter. The Paschen's curve became wide and shifted to lower pressure with increasing the diameter. The reduction area of hollow anode caused dens and luminous intensity of plasma to occur in the negative glow region. Increasing the diameter resulted in decreasing the temperature and density of electron.
\end{abstract}

KEYWORDS: Hollow Anode; Argon Glow Discharge; Double Probes; Plasma Parameters; Negative Glow Region; MATLAB program.

\section{INTRODUCTION}

Experimental and theoretical studies of hollow anode glow discharge show that the variation of hollow inter diameter is an important factor that affects the plasma parameters, and especially in the negative glow region. Miljevic (1985) obtained highly ionized plasma using hollow anode and concave cathode. The density of high-energy electrons increases indicating that the hollow anode discharge is effective if the dimension of the hollow anode is small. Anders et al (1996) used hollow anode discharge with very small anode area, a positive anode voltage drop of 30-40 V. They observed that the increase in the thickness of anode sheath leads to ignition of dense plasma in front of anode. Shigeru (Yokota et al, 2004) studied the effect of low and high magnetic flux density on the plasma density, ion sheath and electron sheath. Chine and Ricky (2005) investigated hybrid evaporation glow discharge, which contains a hollow anode with diameter $6 \mathrm{~mm}$. Their results show resemblance to hollow anode glow discharge and the anode fall is faster than the general glow discharge.

Tutyk (2008) concluded that at pressure in the range of (101000) $\mathrm{Pa}$, the steepness (I) of discharge decreases when the pressure rises and increases with the increase of the HA diameter. It is also shown that the steepness strongly depends on the type of puffing gas. A study on laser induced fluorescence of the ferroelectric plasma source assisted hollow anode discharge developed by (Vekselman et al, 2009), they have shown that plasma filling of the hallow anode cavity occurs and the expansion of the plasma causes increase of the plasma density. Savkin et al (2010) used longitudinal hollow anode and several cathode materials $(\mathrm{Cu}, \mathrm{Mo}, \mathrm{Au})$ and argon as operating gas to generate a mixer of gaseous-metal ion beams. A hollow anode discharge sources developed by (Kang

* Corresponding author

This is an open access under a CC BY-NC-SA 4.0 license (https://creativecommons.org/licenses/by-nc-sa/4.0/) et al, 2013) were used to produce supersonic plasma jet with high density of the high-energy electrons. Gleizer et al, (2014) used a hollow anode discharge at low-pressure to generate a high-current electron beam. It was found that the rising time decreases monotonically by increasing the pressure. Timoshenko et al, (2015) presented plasma parameters of nonself-maintained discharge with hollow anode to increase the ion flux, this type of discharge may be effectively used for ion pumping film deposition. Al-Hakary (2016) studied the effect of direct current hot cathode and hollow anode argon glow discharge plasma at different pressures in the range $0.05-0.8$ mbar on some plasma parameters, ion temperature, degree of ionization and electron energy distribution function as well as some characteristics of glow discharge.

The present study investigates experimentally the effect of inner hollow anode diameter on the breakdown voltage of discharge (Paschen's curve) and its effect on some plasma parameters (electron temperature and its density) in the negative glow region of discharge at different argon pressures.

\section{EXPERIMENTAL EQUIPMENT}

The discharge tube is a cylindrical Pyrex glass of $5 \mathrm{~cm}$ diameter and $25 \mathrm{~cm}$ length as shown in figure 1 . The tube is opened in both sides in order to move the electrodes and adjust the gap between electrodes to obtain best homogenous discharge. The tube has two pipe connections. The first pipe is connected to a valve which involves two apertures one of them is for gas inlet and the other is for rotary vacuum pumping. The second pipe has a diameter $2.95 \mathrm{~cm}$ and $2.5 \mathrm{~cm}$ length for inserting the probe. Hollow cylindrical anodes are made of brass metal of inner diameter values of $(10,15,20,25,30,35$ and $40 \mathrm{~mm})$, all anodes have the same depth, thickness and length $(30 \mathrm{~mm}, 5$ 
$\mathrm{mm}$ and $40 \mathrm{~mm}$ ) respectively. Circular cathodes are also made of brass disks plates with thickness of $5 \mathrm{~mm}$ and $20 \mathrm{~mm}$ in diameter. Cathode and hollow anodes are connected to external electric circuit by the copper rod fixed on the backside of electrode and pass through a cylinder rubber to prevent leakage. Rotary vacuum pump (Trivac E2, Pr. Nr. 140000, Lybold), is used to evacuate the discharge tube to an ultimate pressure, this in turn is connected to the discharge chamber on one side of cross shaped unit pipe. The pressure was measured by thermocouple vacuum gage (Thermovac TM21) which was connected to one side of this unit pipe. Double probes are made from the alloy of (Nickle- Crome) wire of $0.25 \mathrm{~mm}$ in diameter and $6 \mathrm{~mm}$ in length separated by a distance of $(4 \mathrm{~mm})$. The probes are fixed in a capillary tube of glass and covered by a plastic insulator except at the probe tip. They are inserted within the negative glow region and are used to measure the electron temperature and electron density in the negative glow region. Direct current voltage has been applied to the electrodes from a variable DC power supply of range $(0-6000)$ volt (3B power supply U21060), sufficient to produce breakdown. The nonlinear protective resistor (lamp) is used to control the discharge current and produce avoidable stream to pass through the chamber to make spark breakdown [77s], The probes sweep voltage is obtained from a variable AC power supply (Lybold Didactive GMHZ 52135 WR00001888, 250-50) HZ, biasing the probes at about 12 Volt peak value, using isolating transformer. The probe current is sampled across a direct resistance $(100 \mathrm{k} \Omega)$. The capacitor (C50 SAMER 29539), was used to reduce the phase difference in $\left(\mathrm{I}_{\mathrm{p}}-\mathrm{V}_{\mathrm{p}}\right)$ characteristic of probe caused by astray capacitances in the circuit. The (X-Y) recorder (oscilloscope) traces the I-V characteristic of the probe, so the $\mathrm{x}$-axis recorded the voltage on the probe, while the current through the probe is recorded on the y-axis, as the current passed through the resistance of value $(100 \mathrm{~K} \Omega)$. Data for the probe I-V characteristics are acquired using the computer sound card as the data acquisition device in conjunction with special MATLAB program.

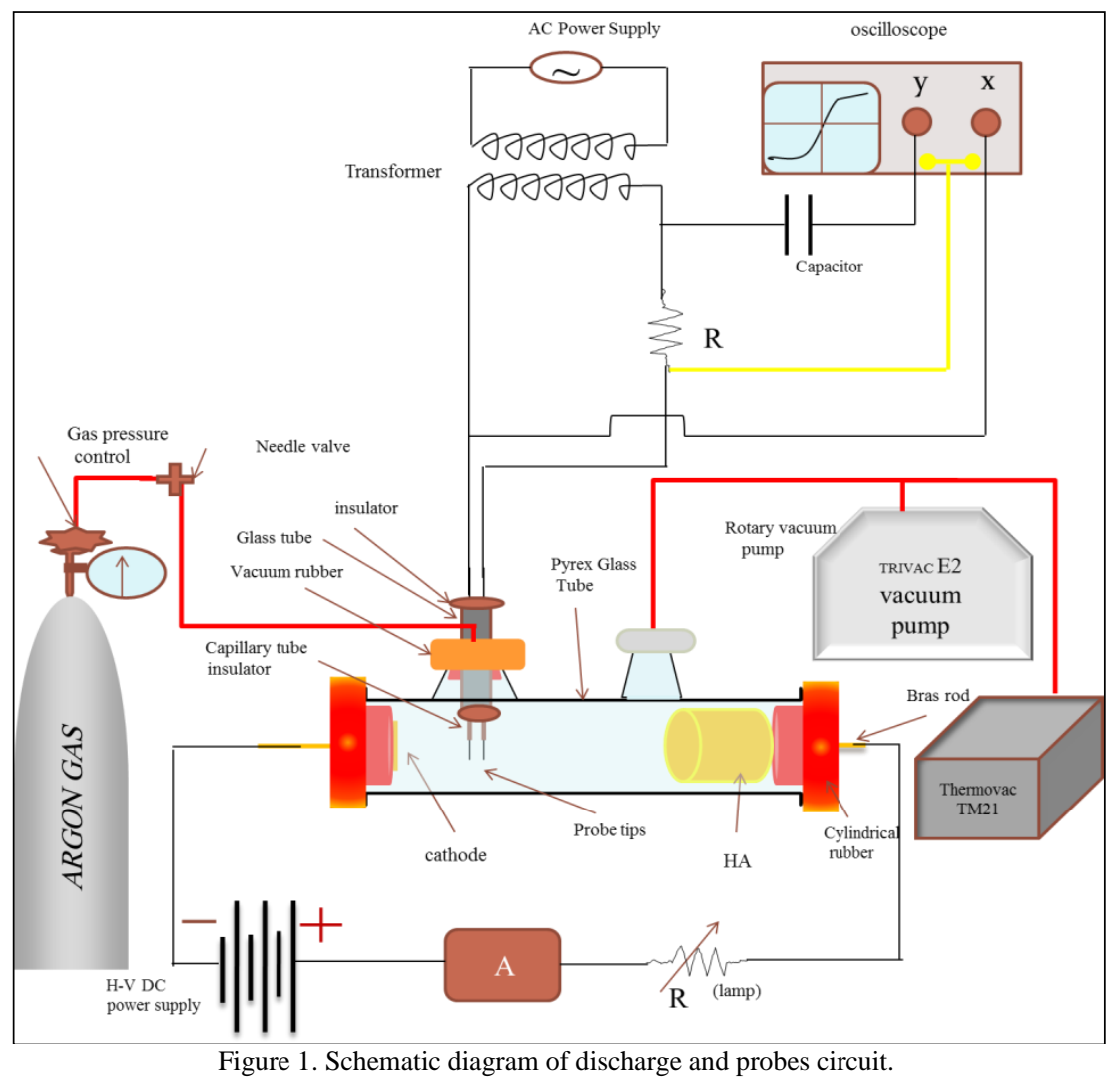

\section{RESULTS AND DISCUSSION}

This section will be divided into two parts: The first will describe the results of breakdown voltage analysis. The second will discuss the plasma parameters results.

3.1 BREAKDOWN VOLTAGE: The experiments were conducted firstly to measure the breakdown voltage at different value of pressure ranged from $2.2 \times$ to $3 \mathrm{mbar}$ ). The processes are repeated for different diameters of hollow anode using constant cathode disc of radius $20 \mathrm{~mm}$ to investigate the Paschen's curve under the influence of hollow anode geometry. The experimentally obtained Paschen's curves at different diameters are close to the well-known Paschen's law. These are shown in figures 2 to 8 . The results indicate that higher voltage values are needed at low and high (Pd). It is interesting to note that these Paschen curves tend to show some widening when the diameter of hollow anode is increased. This agrees with the results of some recent studies (Teo Yong and Teng, 2010) where similar widening was reported.

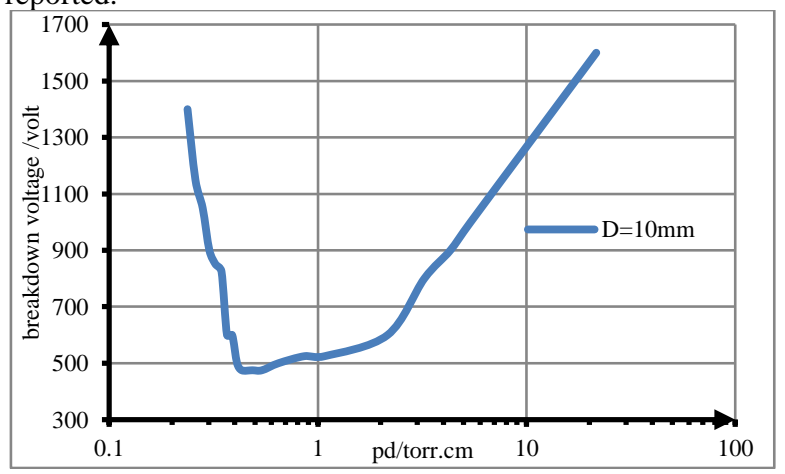

Figure 2. Paschen's curve for diameter (10mm) 


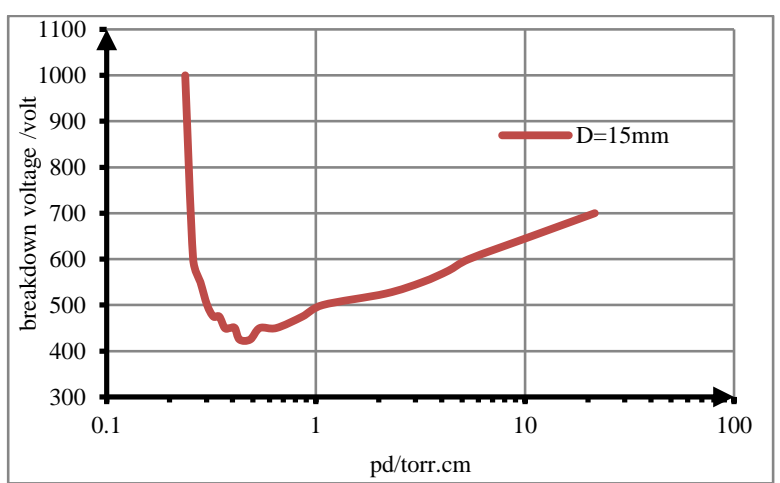

Figure 3. Paschen's curve for diameter (15mm)

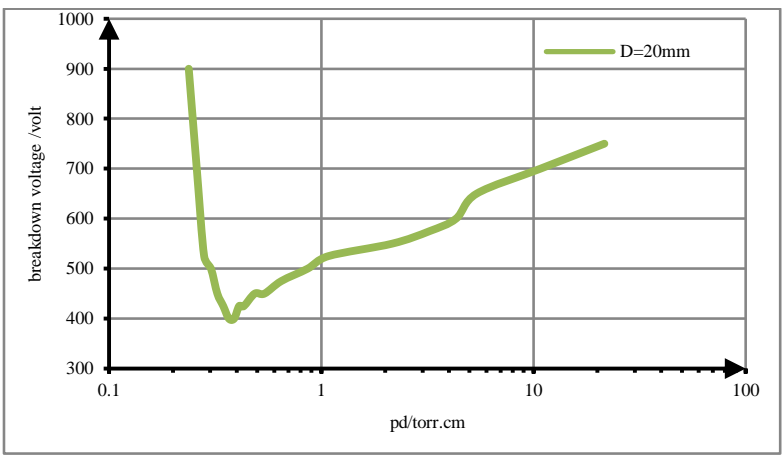

Figure 4. Paschen's curve for diameter (20mm)

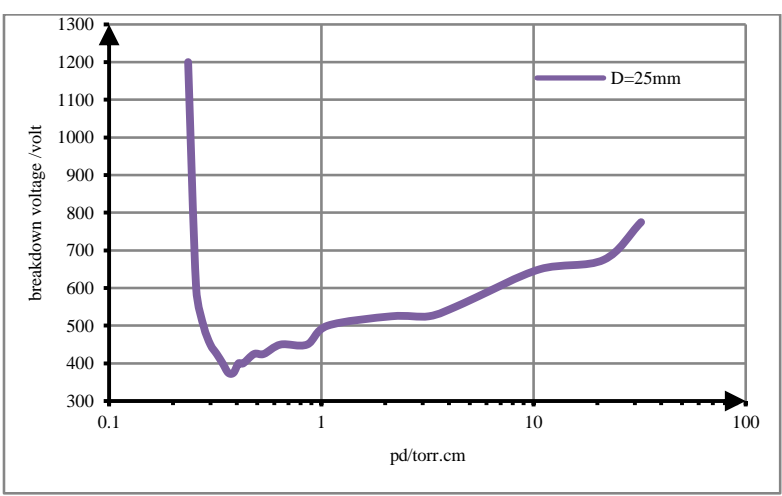

Figure 5. Paschen's curve for diameter (25mm)

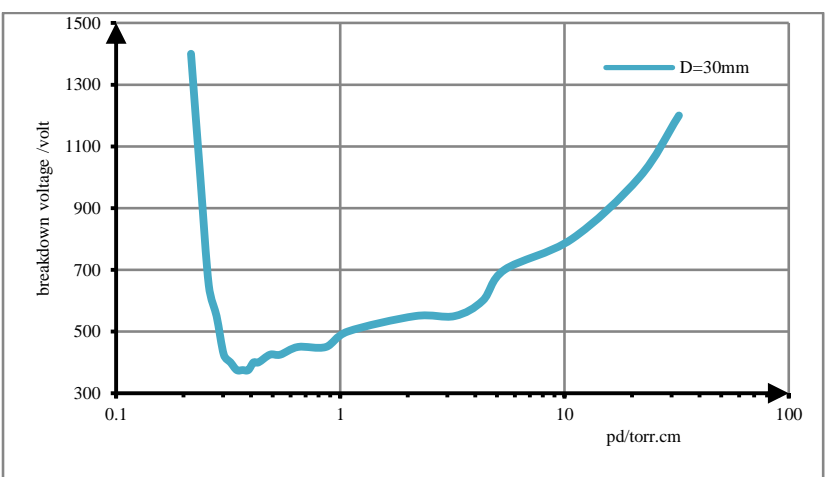

Figure 6. Paschen's curve for diameter (30mm)

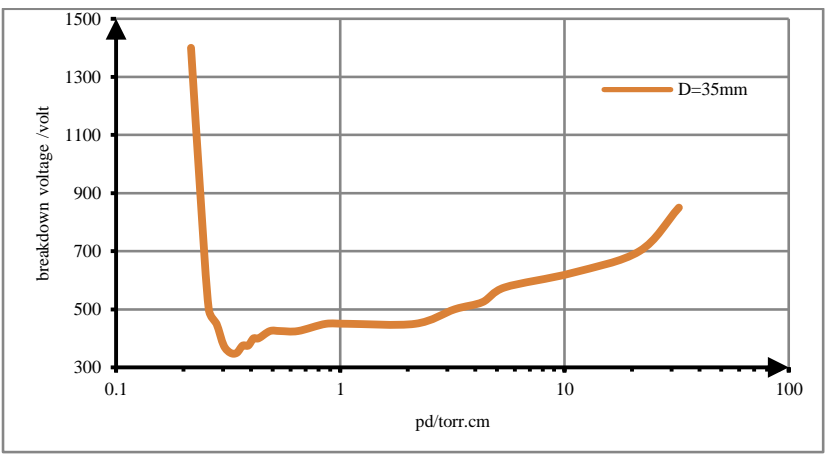

Figure 7. Paschen's curve for diameter (35mm)

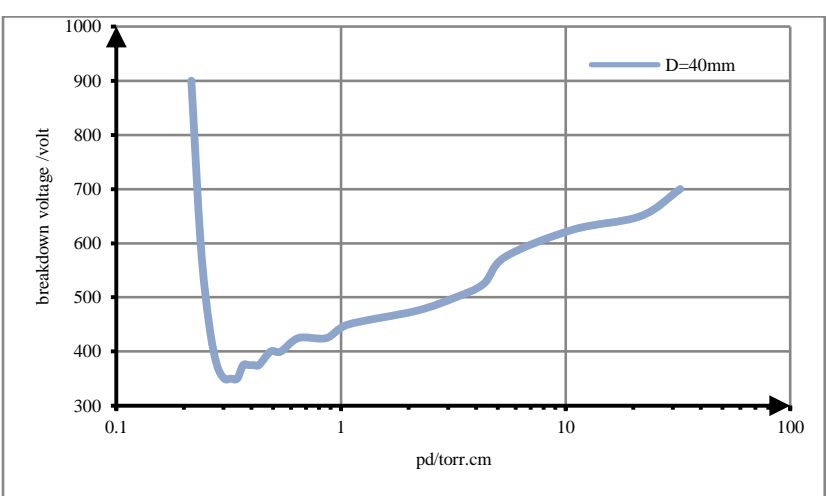

Figure 8. Paschen's curve for diameter $(40 \mathrm{~mm})$

The effect of increasing internal area of hollow anode resulted in the increase of the second Townsend coefficient. This is due to the increase of probability of creating secondary electrons. Consequently, the electron current flux density is also increased causing reduction of the breakdown voltage. It is observed that the breakdown voltage decreases according to the increase of the diameter of hollow anode, as shown in three-dimensional figure 9 .

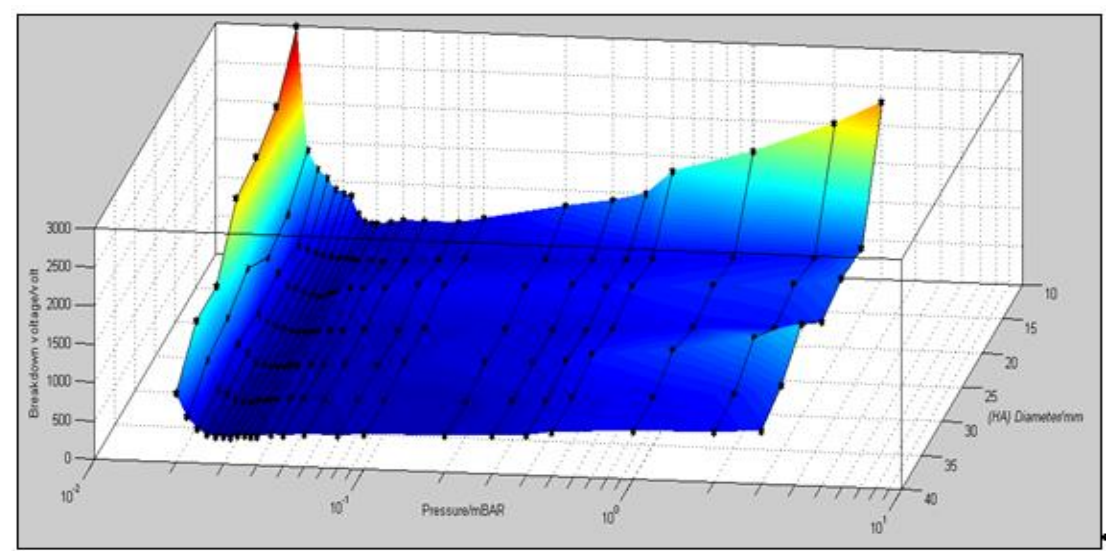

Figure 9. Breakdown voltage versus hollow anode diameter at different values of gas pressures 
Figure 9, indicates that at any fixed value of pressures, the breakdown voltage decreases due to enhancing hollow anode diameter which are represented in the figure by dark lines. This is attributed to increasing the local ionization collisions in the anode sheath, which leads to increase the second Townsend coefficient.

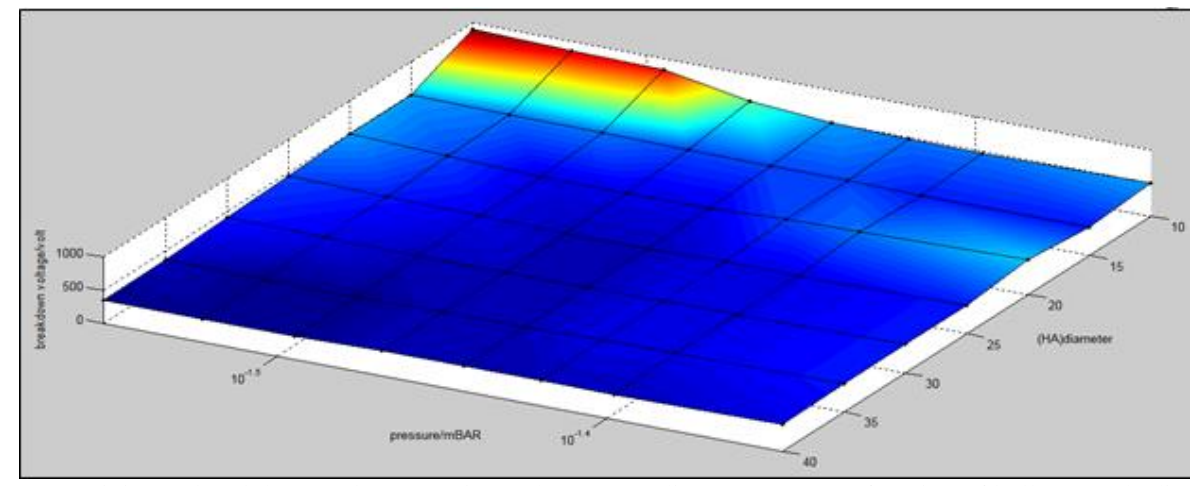

Figure 10. Minimum breakdown voltage (Vmin) and corresponding pressure as a function of hollow anode diameter.

Figure 10; indicates that the bottom of the valley of the curve tends to shift toward the lower breakdown voltage and lower pressure as the hollow anode diameter increases. This means that the minimum breakdown voltage is a function of second Townsend coefficient resulting reduction of the breakdown voltage by using a cylindrical hollow anode with large diameter as shown in figure 11.

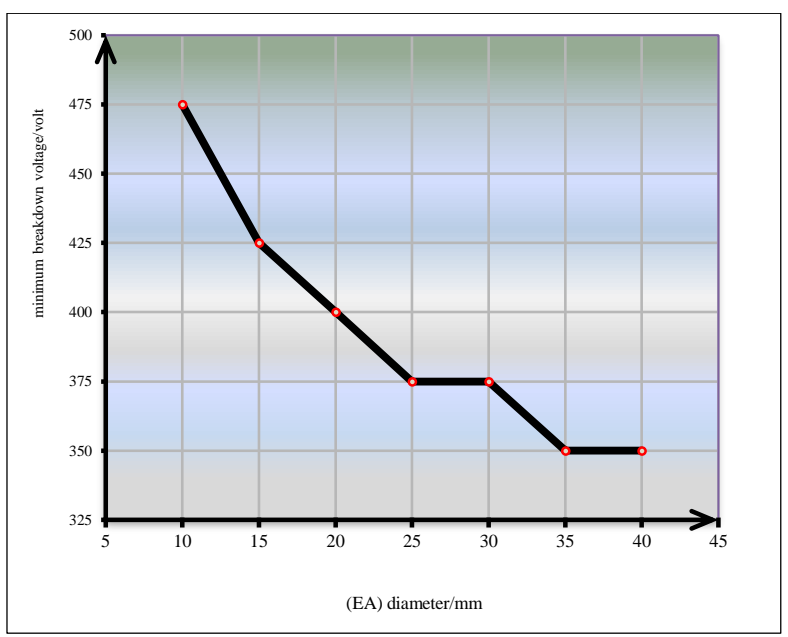

Figure 11. Minimum breakdown voltages versus anode diameters

On the other hand, increasing anode diameter will enhance the number of gas atom inside the hollow anode. Accordingly, the radial electric field per atoms $\left(\mathrm{E}_{\mathrm{r}} / \mathrm{N}\right)$ decreases. Therefore, it becomes difficult for electron to make ionizing collision with neutral atoms as well as to impact the wall side of anode at same pressure. The process thus needs less pressure to get minimum breakdown voltage as shown in figure 12 .

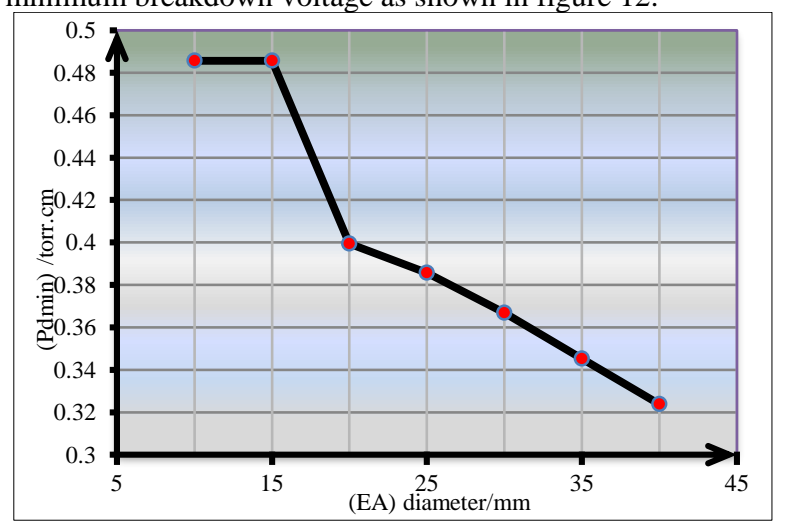

Figure 12. (Pdmin) versus hollow anode diameter
3.2 PLASMA PARAMETERS: The second part of our study deals with electron density and its temperature in the negative region of glow discharge. This region is the second large luminous region after positive column. For this purpose Langmuir cylindrical double probes inserted in the center of this region and fixed along the discharge axis. The double probes $\left(I_{p}-V_{p}\right)$ characteristics as shown in figure 13, were analyzed using computer MATLAB program written for obtaining the electron density and its temperature under the effect of hollow anode geometry.

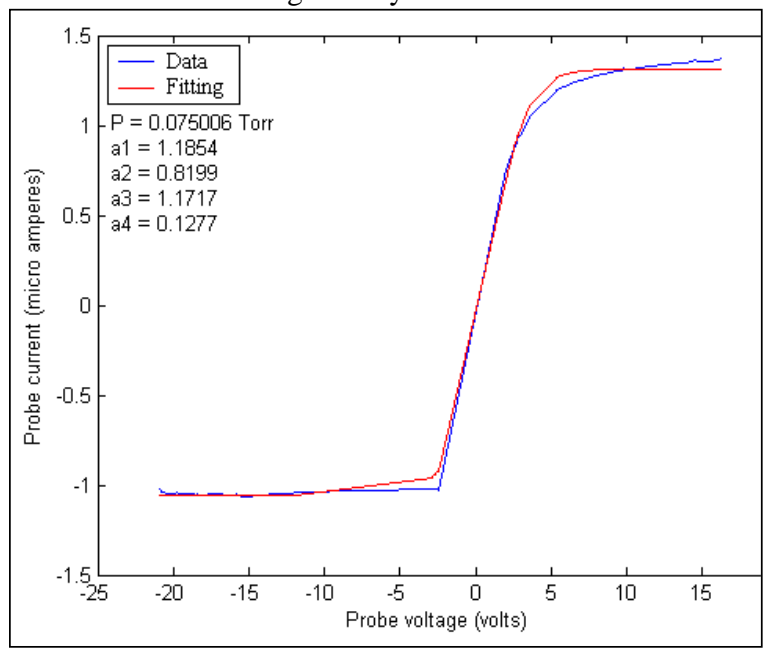

Figure 13. Double probe (Ip-Vp) characteristic at center of negative glow region, $\mathrm{Id}=1.850 \mathrm{~mA}$ )

Electron temperature is one of the most important parameters, which depends on the ionization collisions and transfers of energy (Andre el al, 1995). The electron temperature calculated from double probe $\left(\mathrm{I}_{\mathrm{p}}-\mathrm{V}_{\mathrm{p}}\right)$ characteristics at different pressure for each hollow anode is shown in figures 14 and 15. 


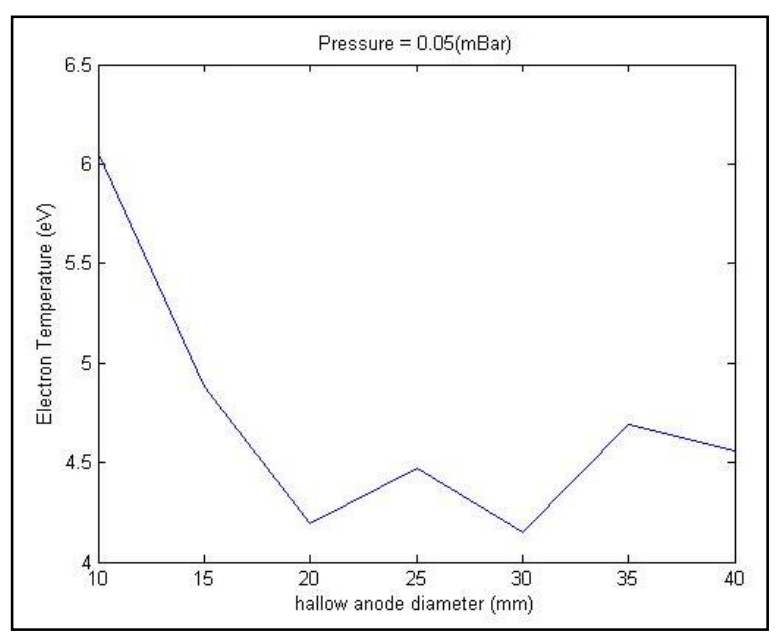

Figure 14. Electron temperature varsuse hollow anode diameter at Pressure $0.05 \mathrm{mbar}$.

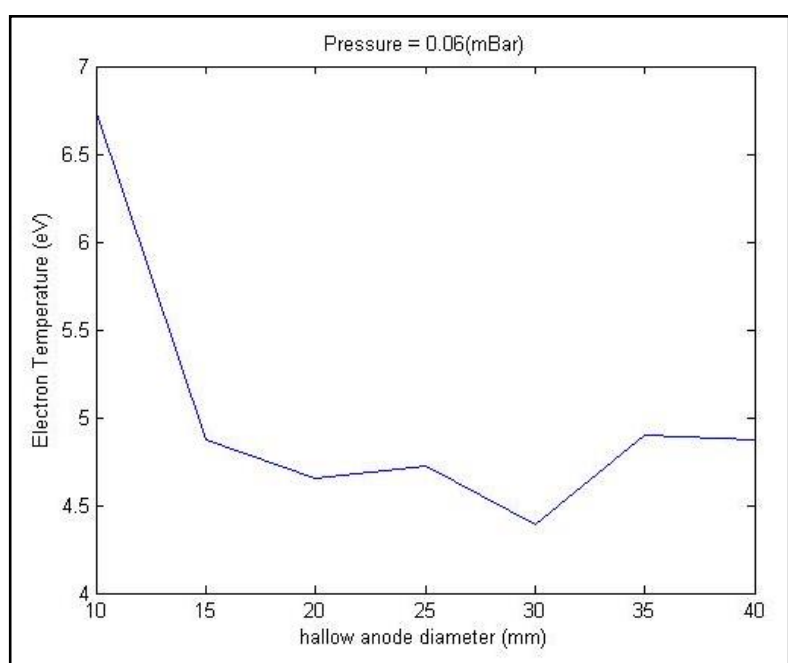

Figure 15. Electron temperature varsuse hollow anode diameter at Pressure $0.06 \mathrm{mbar}$

It is obvious from the figures 14 and 15 that by increasing hollow anode diameter at constant depth, the electron temperature will decrease. This behavior is due to the reduction of length of negative glow region and consequently reduction of luminosity in aforementioned region. Also, it is attributed to the decreasing of the number of electron excitation and ionization collisions with the other plasma particles in this region. These two effects result in decrease in the electron temperature.

Electron density is an important parameter in plasma processing because the efficiency of the processes occurring in the plasma and their reaction rates are generally depended directly on the density of the charged particles (Grill, 1993). This parameter is plotted as a function of the hollow anode diameter at different pressure as shown in figures 16 and 17.

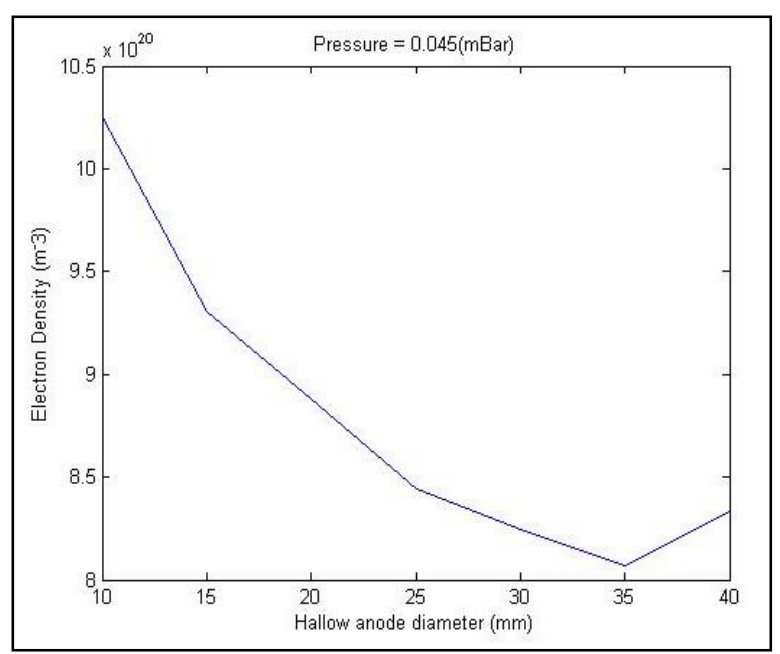

Figure 16. Electron density versus hollow anode diameter at pressure $0.045 \mathrm{mbar}$

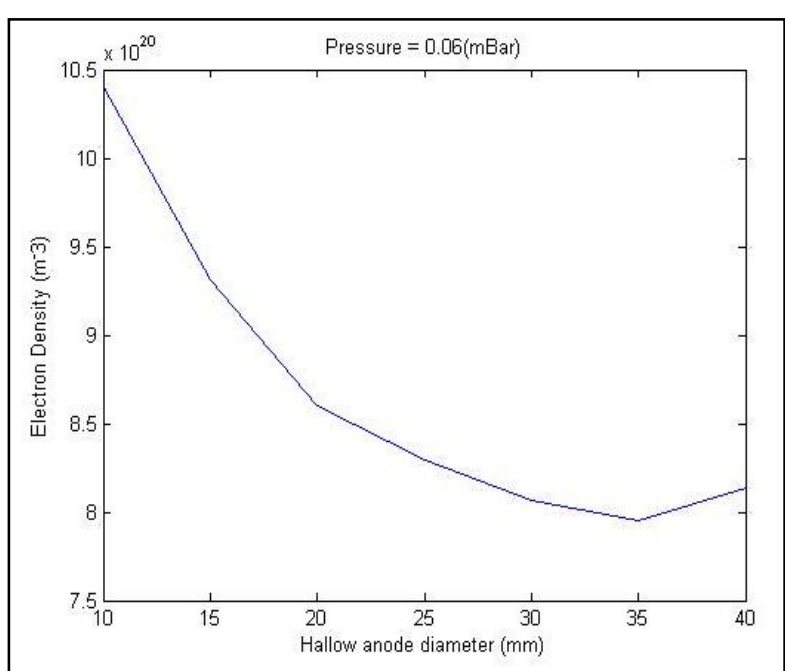

Figure 17. Electron density versus hollow anode diameter at pressure $0.06 \mathrm{mbar}$

It is obvious from the above figure 17, that the electron density decreases because of the increase of hollow anode diameter. This effect is attributed to the decrease of the number of excitation and ionization collisions.

\section{CONCLUSION}

A comprehensive experimental study of the hollow anode DC glow discharge from the negative glow and Positive column regions in argon gas has been performed. The Pashence's curve has been measured as a function of hollow anode geometry for different argon gas pressures. In parallel to that, measurements of some important plasma parameters in the negative glow region of discharge have been done. The most important conclusions deduced from this work can be summarized as follows:

1. Measured Pashence's curve close to the well-known theoretical Pashence's law.

2. It is possible to minimize the breakdown voltage by increasing the diameter hollow anode.

3. Increasing the hollow anode diameter causes the Paschen's curve to become wide and shift to lower pressure values.

4. A more dens and luminous intensity of plasma occurs in the negative glow region by reducing the area of hollow anode.

5. A Computer MATLAB program which is made to estimate the $T_{e}$ and $n_{e}$ gave reasonable results of plasma parameters. 
6. Electron temperature and its density decreases due to the increase of diameter of hollow anode.

\section{REFERENCES}

Al-Hakary, Sh. Kh. (2016). Effect of Pressure and Hot Filament Cathode on Some Plasma Parameters of Hollow Anode Argon Glow Discharge Plasma, American Journal of Modern Physics. Vol. 5, No. 3, 2016, pp. 30-38.

Anders, A., Newman, N., Rubin, M., Dickinson, M., Thomson, A., Jones, E., Phatak P. and Gassmann, A. (1996), Hollow Anode Plasma Source for Beam Epitaxy of Gallium Nitride. Lawrence Berkeley National Laboratory, Berkeley, CA 94720.

Andre, A. and Simone, A. (1995). Working Principle of the HollowAnode Plasma source, Plasma Sources Science Technology, 4: p. 571-575

Glaizer, J. Z., Yarmolich, D., Krokhmal A., Krasik, Y. E., and Felsteiner J. (2005). Optimization of a Low-Pressure Hollow-Anode Electrical Discharge for Generation of High-Current Electron Beams. Journal of Physics D: Appl. Phys. 38. 2.

Glaizer, J. Z., Yarmolich, D., Krokhmal, A., Krasik, Y. E., and Felsteiner, J. (2005). Optimization of a Low-Pressure Hollow-Anode Electrical Discharge for Generation of High-Current Electron Beams. Journal of Physics D: Appl. Phys. 38. 2.

Grill, A. (1993), Cold plasma in materials fabrication from fundamentals to applications, Institute of electrical and electronic engineers, Inc., New York, USA
Kang, I. J., Cho, S. G., KeunBae, M., KiuJoo, S., Kim, J. W., Kim, H. J., and Chung, K. S. (2013). Design of a Hollow-Anode Discharge Source for Generation of Supersonic Plasma Jets. APS division of Plasma physics, Vol. 58, No.16.

Miljevic, V.I. (1985). Characteristics of the Hollow Anode IonElectron Source. J. Appl. Phys. 57, 4482

Savkin, K.P., YuSkov, Yu. G. Nikolaev, A.G., Oks, E.M., and Yushkov, G. Y. (2010). Generation of Gaseous and Metal Ions in Vacuum Arc Discharge with the Longitudinal Hollow Anode in a Magnetic Field. TUBITAK and RFBR under the joint Turkish-Russian research program (Grant Nos. 108M394 and RFBR-09-08-91219- CT a).

Teo Yong, S. and Teng, P.I., (2010). Plasma Physic: Paschen Curve, Measure the Breakdown Voltages $\mathrm{U}_{\mathrm{br}}$ at Varying Electrode Gap Distance d under a fixed pressure $\mathrm{P}$ of air.

Timoshenkov, O., Misiruk, I.O., Taran, V.S., and Garcusha, I.E. (2015). Non-self-maintained discharge with a hollow anode: characteristics and application. $32^{\text {nd }}$ ICPIG, Iași, Romania.

Tutyk, V.A. (2008). Low-Vacuum Gas-Discharge Electron Guns on the Basis of High-Voltage Glow Discharge. Problems of atomic science and technology. Plasma Physics,14, 156158.

Yokota, S., Komurasaki, K., and Arakawa, Y. (2014). Plasma Density Fluctuation inside a Hollow Anode-Layer Hall Thruster.

Vekselam, V., Glaizer, J., Yatom, S., Yarmolich, D., Gurovich, V. Tz., Bazalitski, G., Krasi, Y. E. and Bernshtam, V. (2009). Laser Induced Fluorescence of the Ferroelectric Plasma Source Assisted Ohollow anode discharge" Physics of Plasmas, 16. 113504 . 